\title{
SCANNING-ION MICROSCOPY WITH POLARIZATION ANALYSIS (SIMPA)
}

\author{
N. J. Zheng and C. Rau
}

Department of Physics and Rice Quantum Institute, Rice University, Houston, TX 77251.

\begin{abstract}
We have developed a novel, high-resolution magnetic imaging technique, scanning-ion microscopy with polarization analysis (SIMPA). In SIMPA, a highly-focused, scanning $\mathrm{Ga}^{+}$ion beam is used to excite spin-polarized electrons at surfaces of ferromagnetic materials. By measuring the intensity and the spin polarization of the emitted electrons using a newly developed, compact Mott polarimeter, topographic and magnetic images of magnetic structures are obtained. We report on first SIMPA studies on single-crystalline Fe samples.
\end{abstract}

\section{INTRODUCTION}

Quite recently, secondary electron emission (SEE) has attracted, particularly in combination with analysis of the electron spin polarization (ESP), broad scientific attention. This has been shown by the implementation of secondary electron microscopy with polarization analysis (SEMPA) [1] and of spin-polarized electron emission spectroscopy (SPEES) [2]. Electronand ion-induced SEE provides a high electron yield which is needed for efficient, highresolution topographic and spectroscopic imaging of surfaces. Besides this, there are two addition features that show the great scientific and technological potential of the use of SEE for the topographic and magnetic imaging of surfaces. Firstly, the ESP of low-energy electrons ( $<10 \mathrm{eV})$ emitted from ferromagnetic surfaces materials is enhanced by a factor of two or three as compared to the average magnetization of the material (valence band polarization) [3]. This, when utilized for magnetic imaging, will enhance the image contrast by a factor of four to six. Secondly, the inelastic mean free path of these low-energy electrons in $3 \mathrm{~d}$ transition metals is, as recently found [4], only of the order of one or two monolayers, which is much shorter than the values published $(3 \mathrm{~nm}-5 \mathrm{~nm})$ in the past decades. It is especially this characteristic that makes the use of SEE very attractive for high-resolution, topographic and magnetic imaging of bulk and ultrathin film materials.

With growing interest in magnetism in thin film and multilayer-structured systems, especially with respect to their potential technological applications, a deeper and more fundamental understanding of the influence of the geometrical and chemical micro-structure on surface and interface magnetic properties becomes an area of revived scientific research.

In this paper, we report on a novel, high-resolution magnetic imaging technique, scanningion microscopy with polarization analysis (SIMPA). In SIMPA, a micro-focussed ion beam is used to excite electrons at the surface and in deeper-lying layers of a magnetic material. As shown in several recent publications, these ion-induced electrons are spin-polarized $[2,5,6]$. An enhancement of the ESP of the emitted low-energy electrons is found similar to that detected in electron-induced SEE from magnetic materials. In addition, it is found that the surface sensitivity can be varied either by modifying the ion beam energy [5] or the incidence angle of the ions $[6,7]$, 
thus offering a promising way to study layer-dependent, microstructural magnetic properties at surfaces of bulk and thin film materials.

Our motivation for the work, presented here, was to develop a new element-specific and spin-sensitive, magnetic and nonmagnetic imaging technique which allows us to vary the probing depth from the topmost surface to deeper layers. In addition, by varying the intensity of the incident ions, SIMPA offers the capability to use the technique not only for imaging but also for depth-profiling and for the etching of 3D patterns. To this, we note that the technique of secondary ion mass spectroscopy (SIMS) is already widely recognized as a reliable technique for chemical depth profiling [8].

By measuring the ESP of emitted Auger electrons, SIMPA offers an additional attractive feature which allows the element-specific imaging of surface magnetic properties. We note that, as regards this feature, technical constraints as regards a timely compilation of the data have yet to be overcome. At present, a combination of SIMPA and SIMS seems to be more advantageous than to use solely SIMPA for scanning Auger electron imaging in order to be able to understand the effects of chemical composition on micro-structural magnetic properties.

We note that there is evidence that ion beam sputtering does not alter magnetic properties during depth profiling. Kirschner et. al. [9] recently measured the ESP of secondary electrons emitted from an oxidized Fe crystal as function of oxide film thickness. From the results of their experiments, they conclude that ion beam sputtering does not modify the magnetic properties of the Fe crystal.

\section{EXPERIMENTAL}

For our studies on topographical and magnetic surface imaging, we used an ion gun system with a Ga+ liquid metal (LM) ion source which is installed in an ultrahigh vacuum system with base pressure of $5 \times 10^{-11}$ mbar. This system is utilized to provide a micro-focussed (minimum beam spot size: $50 \mathrm{~nm}$ ) $\mathrm{Ga}^{+}$ion beam for the studies presented here. The beam energy can be varied from 7 to $30 \mathrm{keV}$. The entire experimental set-up ( 2 interconnected UHV systems) is suspended by six air springs to isolate floor vibrations during imaging at high resolution. The two interconnected UHV systems are used for sample preparation, thin film growth, sample characterization (RHEED, AES, etc.) and for imaging studies. A 48-inch long mechanically and magnetically coupled linear/rotary feedthrough is used for in situ sample transfer between the two UHV chambers. This manipulator is part of a fast entry-air lock system which enables us to load samples from ambient pressures first into a pressure stage, working at $10^{-7} \mathrm{mbar}$, and then into the UHV system.

The SIMPA setup, together with a CMA Auger spectrometer and a scanning tunneling microscope is housed in the characterization chamber as shown in Fig. 1. The samples are mounted on a manipulator which is located at the center of the characterization chamber. For the surface imaging, we use $\mathrm{Ga}^{+}$ions incident at an angle of $45^{\circ}$ towards the surface to be imaged. The ion-induced electrons emitted from the sample surface are collected using an extracting lens system which is connected to a Mott polarimeter operating at $20 \mathrm{keV}$. At present, we are analyzing the two in-plane components of the ESP of the collected electrons. For fast adjustment of imaging parameters and precise location of surface areas to be imaged, we use a conventional electron detector and collect secondary electrons which allows for the display of images on a TV monitor at TV rates.

Using a Mott polarimeter, the ESP of electrons is determined by measuring the asymmetry in the $120^{\circ}$-scattering of spin-up and spin-down electrons from a high- $\mathrm{Z}$ material which is due to spin-orbit interaction. Without including instrumental asymmetry, the component $\mathrm{P}$ of the ESP 


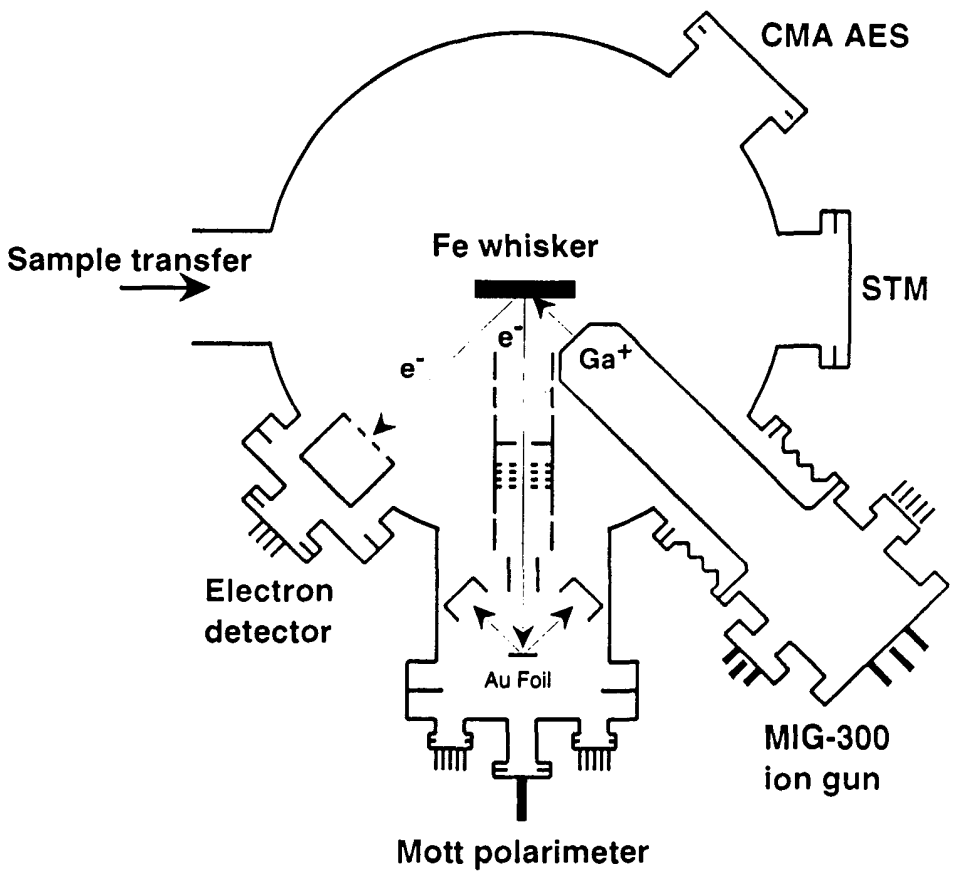

Fig. 1. Schematic of SIMPA set-up.

along the direction normal to the scattering plane is determined by

$$
\mathrm{S} * \mathrm{P}=(1-\mathrm{A}) /(1+\mathrm{A}),
$$

where $S$ is the Sherman function $(\approx 0.1)$ and $A=N_{R} / N_{L}$ is the scattering asymmetry with $N_{R}$ and $\mathrm{N}_{\mathrm{L}}$ being the count rates of electrons detected in the channeltrons $R$ and $\mathrm{L}$, respectively.

The relative inefficiency of electron backscattering requires a longer acquisition time for a magnetic image than for a topographic image. Therefore, our electron extracting lens system for the Mott polarimeter is designed to take advantage of the high ESP and high intensity of lowenergy electrons in SEE from magnetic materials. Our lens design possesses an acceptance angle of $180^{\circ}$ for electrons with energy below $6 \mathrm{eV}$ and of $140^{\circ}$ for electrons with energy above $10 \mathrm{eV}$. These values are determined using a computer (SIMION) program for calculation of trajectories of charged particles. We note that the influence of strong electric fields, applied at the entrance of the extracting lens system, has a negligible effect on the trajectories of the ions used for scanning the surface. For spectroscopic measurements, four retarding grids are incorporated into the lens system. The presence of these grids, however, reduces the transmisivity of the lens system to $64 \%$. Details and performance of the electron polarimeter will be given elsewhere. 
An L-shaped, single crystalline Fe whisker is used as sample for our first SIMPA imaging measurements. The sample surface is cleaned in situ by $\mathrm{Ar}^{+}$sputtering, and the surface cleanness is checked using CMA AES. Residual carbon and oxygen concentrations are less than $2 \%$.

\section{RESULTS AND DISCUSSION}

Figure 2 shows an intensity spectrum of electrons emitted from a polycrystalline Fe surface as a function of applied retarding voltage $(0-30 \mathrm{~V})$. From the results of this experiment, where we used a $12 \mathrm{keV} \mathrm{Ga}+$ ion beam, it is obvious that cascading electrons with energies below $6 \mathrm{eV}$ have a major contribution to the total electron yield being used as signal for magnetic imaging. From this, we estimate collection efficiency of our extracting lens system to be at around $60 \%$.

The inset in Fig. 2 shows the electron yield, $\gamma$, as a function of ion energy $E_{i} \cdot \gamma$ increases from 0.5 at $E_{\mathrm{i}}=9 \mathrm{keV}$ to 1.3 at $\mathrm{E}_{\mathrm{i}}=25 \mathrm{keV}$.

For fast ESP measurements, high electron intensities are required because of the low efficiency of the Mott polarimeter. The statistical error in the ESP is determined by

$$
\Delta \mathrm{P}=(1 / \mathrm{F} \mathrm{N})^{1 / 2},
$$

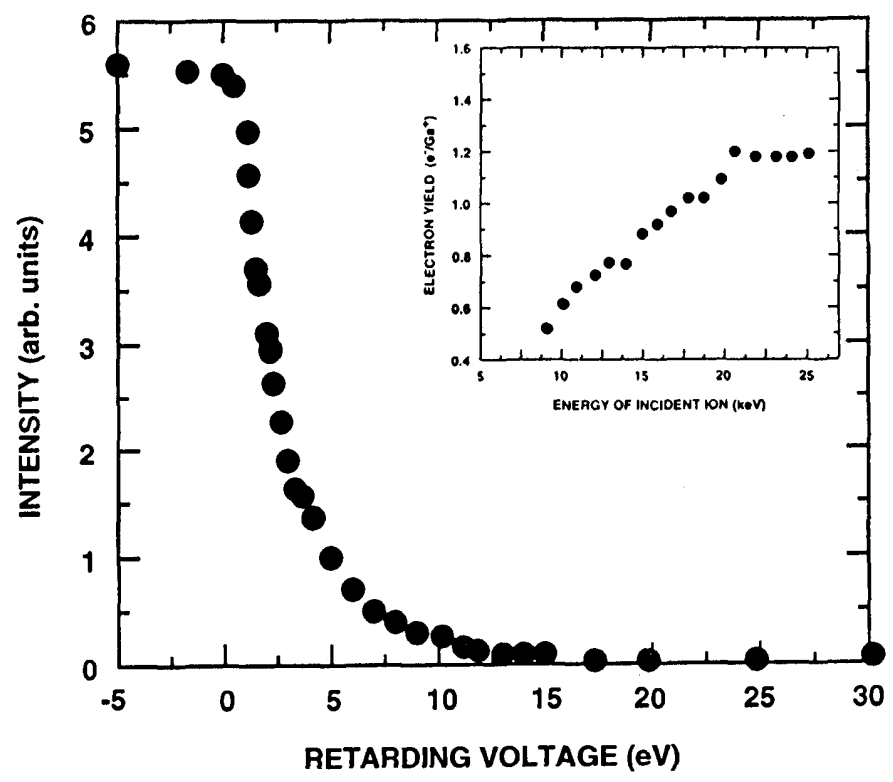

Fig. 2. Ion-induced electron emission spectrum of an Fe surface as a function of retarding voltage with incident beam energy of $12 \mathrm{keV}$. The inset shows the electron yield as a function of the energy of incident ions. 
where $\mathrm{F}$ is the figure of merit of the spin polarimeter $\left(\approx 10^{-4}\right)$ and $\mathrm{N}$ is the number of electrons to be analyzed. To achieve a statistical error smaller than $5 \%, N$ needs to be $\approx 10^{7}$. In our present, we use an ion beam current of less than $1 \mathrm{nA}$ for the magnetic imaging and record one image (256 $\mathrm{x}$ 256 pixels) in about 100 s.

The upper section of Figure 3 shows a magnetic (a) and a topographic (b) image of a selected area $(500 \mu \mathrm{m} \times 500 \mu \mathrm{m})$ of the surface of the L-shaped Fe whisker. The two images are obtained simultaneously. The incident beam energy and the beam current are $15 \mathrm{keV}$ and $0.5 \mathrm{nA}$, respectively. The magnetic image clearly shows two large magnetic domains. It appears that two small topographic defects (small black spots) displayed in Fig. 3 (b) pins the domain walls recorded in the magnetic image which is shown in Fig. 3(a). The lower sections of Fig. 3(a) and 3(b) display two line profiles of the magnetic signal and of the total electron intensity along the traces marked in the upper section of Fig. 3. From this, it is obvious that the topographic line scan displays a
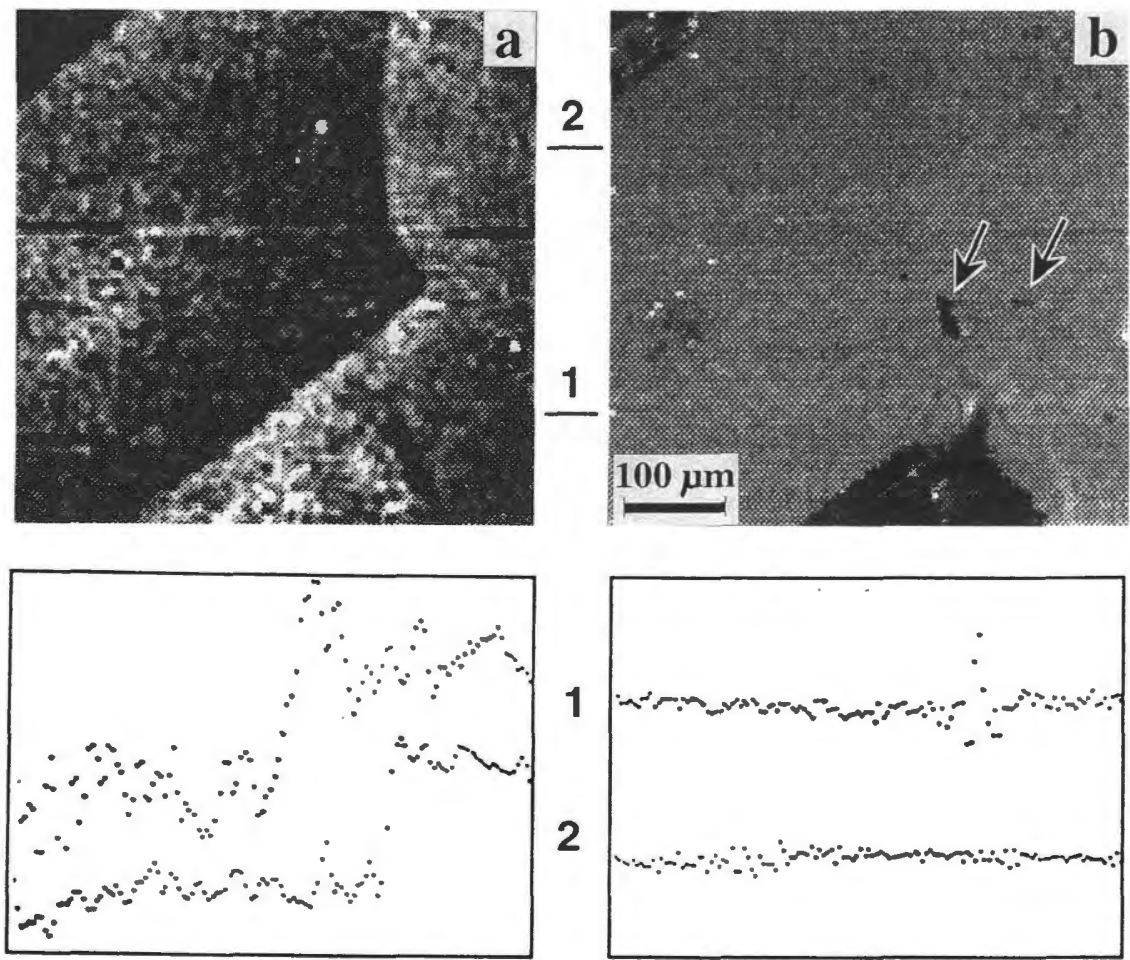

Fig. 3. Simultaneously-acquired SIMPA images of (a) magnetic and (b) topographic structure of an L-shaped single crystal Fe whisker. The image size is $500 \mathrm{X} 500 \mu \mathrm{m}^{2}$. The bottom section shows the line profiles of magnetic and intensity signals along the traces marked in the upper section, respectively. 
smooth surface, and that the magnetic line scan displays ESP values which are abruptly changing from $-30 \%$ to $+30 \%$ as expected when the ion beam scans across a magnetic domain wall. This directly show the power and applicability of SIMPA for magnetic domain imaging.

\section{CONCLUSION}

We have presented first domain images of the surface of a single crystal Fe whisker using a new technique for domain imaging. SIMPA is a new novel and very promising technique which offers many potential applications such as the use of a variable probing depth, magnetic depth profiling, element-specific chemical and magnetic imaging, and etching of magnetic 3D patterns.

\section{ACKNOWLEDGMENT}

We thank H. P. Oepen for many exciting discussions and for providing us an $\mathrm{Fe}$ whisker sample.

\section{REFERENCES}

1. K. Koike and K. Haykawa, Jpn. J. Appl. Phys. 23, L187 (1984); J. Unguris, G. G. Hembree, R. J. Celotta and D. T. Pierce, J. Microsc. 139, SRP1-2 (1985); H. P. Oepen and J. Kirschner, J. Phys. (paris) Colloq. 49, C8-1853 (1985).

2. C. Rau, K. Waters and N. Chen, Phys. Rev. Lett. 64, 1441 (1990).

3. J. Unguris, D. T. Pierce, A. Galejs and R. J. Celotta, Phys. Rev. Lett. 49, 72 (1982).

4. D. L. Abraham and H. Hopster, Phys. Rev. Lett. 58, 13 (1987).

5. J. Kirschner, K. Koike and H. P. Oepen, Vacuum 41, 818 (1990).

6. C. Rau, N. J. Zheng and M. Lu, J. Magn. Magn. Mat. (1993), in print.

7. N. J. Zheng and C. Rau, J. Vac. Sci. Technol. (1993), in print.

8. D. S. McPhail, in Secondary Ion Mass Spectrometry, edited by J. C. Vickerman, A. Brown and N. M. Reed (Oxford University Press, Nèw York, 1989), p. 105.

9. J. Kirschner, K. Koike and H. P. Oepen, Phys. Re. Lett. 59, 2099 (1987). 